\title{
UNA APROXIMACIÓN TEMPORAL A LAS PARTÍCULAS MODALES ALEMANAS
}

\author{
José Javier Martos Ramos
}

\section{INTRODUCCIÓN}

Metafóricamente hablando las partículas modales podrían ser la sal y la pimienta de la lengua alemana. Según en qué cantidad y cómo se utilicen se habrá causado una determinada reacción en el oyente. Por ello antes de abordar el complejo tema de las partículas modales y su relación temporal, considero oportuno plasmar algunas consideraciones y reflexiones sobre las propiedades y características de las partículas relevantes para el análisis propuesto, y su denominación. Estas puntualizaciones, aún pudiendo parecer obvias de manera consciente o inconsciente para investigadores, críticos y estudiantes $^{1}$ de la lengua alemana, son sin embargo un punto de partida para llegar al aspecto temporal de las partículas, además de evidenciar la dificultad que conlleva un manejo correcto de éstas.

En primer lugar y antes de realizar un análisis lingüístico es obligado constatar las dificultades que encuentra el hablante de lengua alemana como L2 para utilizar esta serie de partículas o adverbios modales, que en definición de los lingüistas dotan al discurso de un carácter expresivo. El conflicto lingüístico se genera cuando el hablante de lengua alemana se enfrenta a clases de palabras a las que, o bien no puede asociar un significado, o bien el significado de estas palabras varía en algunos casos aparentemente sin conocer el motivo. El problema se intensifica puesto que al no conocer su significado ni su valor, el hablante no se siente capacitado para usarlas. Normalmente cuando se empieza con el estudio de la lengua alemana son frecuentes frases del tipo Komm doch nach Hause! o ich habe ihn mal gesehen; la mayoría de los problemas del aprendiz de alemán se centran en la comprensión de las partículas. Salvo los alumnos bilingües -que generalmente tampoco están en grado de aclarar y por tanto de transmitir su significado al resto de los alumnos- el valor de las partículas es desconocido en la clase, siendo común oir coletillas como „es difícil traducirlo“ o „no tiene una traducción fija“. A veces ni siquiera el uso de perífrasis ayuda a la comprensión de las partículas; simplemente se responde con un „no significa nada“, se las manda al limbo lingüístico, a la insignificancia, a la inexistencia.

Probablemente estas frases fuera de contexto tienen efectivamente difícil aclaración para un no hablante de alemán como lengua materna. Sin embargo Lew Zybatow propone un texto de fácil comprensión para mostrar que efectivamente algún tipo de significado ya

\footnotetext{
${ }^{1}$ No se incluyen en este grupo a los hablantes nativos de lengua alemana porque, como dice G. Lorenzo (2001:16),....un hablante puede tener pericia absoluta en su manejo siendo totalmente incapaz, sin embargo, de dar explicaciones acerca del conocimeinto que pone en práctica al hablar"“.
} 
El lingüista Harald Weydt ${ }^{5}$, uno de los pioneros en el campo de la lingüística de las partículas $^{6}$ a finales de los 60 y principios de los 70 junto con A. Krivonosov, afirma sobre las veintisiete partículas modales que actúan como tal

„sind unflektierbare Wörtchen, die dazu dienen, die Stellung des Sprechers zum Gesagten zu kennzeichnen. Diese Wörtchen können in gleicher Bedeutung nicht die Antwort auf die Frage bilden und nicht die erste Stelle im Satz einnehmen. Sie beziehen sich auf den ganzen Satz; sie sind im Satz integriert. In anderer syntaktischer Stellung oder anders akzentuiert haben sie alle eine oder mehrere Bedeutungen. In dieser anderen Verwendung gehören sie dann anderen Funktionsklassen an".

Más allá de la mera constatación como hablantes de lengua alemana mencionada anteriormente, Weydt llama la atención sobre otros elementos relevantes en el análisis. Efectivamente conocer el valor que tienen las partículas y saber cuál es la intención del hablante frente al oyente depende en gran medida de la posición que ocupa la partícula en la frase. La pregunta a formular sería qué se entiende por una posición sintáctica diferente. Weydt responde que la posición más común de las partículas, y por consiguiente la norma, es a continuación del verbo. En el siguiente ejemplo que propone Krivonosov se manifiestan las diferentes funciones y significados:

(2a) Warum denn München?

(2b) Also, ich gehe denn jetzt

(2c) Ein Mißverständnis...denn er tut normalerweise sowas nicht

En (2a) se trata de una partícula modal en una frase interrogativa y su función es matizar la actitud del hablante, que en este caso muestra sorpresa. En (2b) el lexema equivale a un adverbio temporal. Se puede sustituir por el también adverbio temporal dann. Y en (2c) se trata de una conjunción causal. Sintácticamente tienen una función diferente. En la primera frase depende de los demás complementos para que pueda tener un valor. En la segunda es un modificador del verbo y en la tercera depende de sí mismo. También se podría formular de otra manera: en la primera frase es posible prescindir de ella, en las dos siguientes no. Este mismo ejemplo remite a otro problema mayor como es el de la homonimia y la polisemia en las partículas modales que ha tratado de manera profunda Krivonosov, pero que por su amplitud no va a ser tratado aquí.

Weydt también afirma en el plano fonológico la carencia de acentuación de las partículas. Esta falta de acentuación es una de la cualidades que no les permitiría ser independiente del resto de los elementos de la frase. Sin embargo añade, que en algunos casos las partículas pueden tener uno u otro significado dependiendo de su acentuación. Tal es el caso de la partícula doch en las siguientes frases:

(3a) Ruf doch án!

(3b) Dóch, er ist gekommen.

\footnotetext{
${ }^{5}$ Weydt, H. (1969): Abtönungspartikel. Die deutschen Modalwörter und ihre französische Entsprechungen, Bad Homburg: Gehlen, pág.68.

${ }^{6}$ Así llama Weydt a esta rama.
} 
En (3a) doch no se acentúa y tiene el valor de partícula modal que refuerza la acción del verbo. En (3b) se acentúa manifestando un valor adversativo.

Helbig/Buscha ${ }^{7}$ afirman también en su gramática al igual que Weydt

„Die Partikeln stellen im Deutschen eine relativ geschlossene Klasse dar, die etwa 40 Wörter umfaßt, die weder deklinierbar noch konjugierbar oder komparierbar sind... (Sie) sind Teile von Stazgliedern. Das unterscheidet sie sowohl von den Adverbien,...als auch von den Modalwörtern...und von den Interjektionen... (Sie) sind auch nicht allein erststellenfähig...(Sie) sind nicht als selbständige Antworten möglich“.

En primer lugar hay que aclarar, qué entienden Helbig/Buscha por palabras modales y qué las diferencia de las partículas. Fundamentalmente la diferencia radica en que las primeras pueden responder a preguntas sobre la frase y las segundas no pueden responder a nada. Proponen como ejemplo la frase Er kommt heute:

(4a) Kommt er heute?

(4b) Wann kommt er heute?

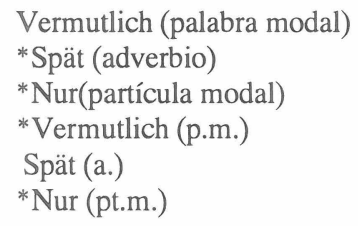

En (4a) spät y nur no son respuestas válidas, y en (4b) vermutlich y nur son las respuestas inválidas. De todo ello se desprende que en ambas frases es imposible utilizar una partícula modal como respuesta. Por el contrario sí es posible utilizar palabras modales y adverbios. Con ello dejan zanjada la cuestión sintáctica que a priori más interesa a Helbig/Buscha.

Erben $^{8}$ las caracteriza como

„emotional-expressive Partikeln, die besonders in umgangssprachlicher Rede geläufig sind und meist emphatisierend wirken, also beinahe als 'Emphasemorpheme' wirken .... die (Modalpartikel) bildet mit der verbalen Aussagekern (Prädikat) eine syntaktische und phonetische Einheit, gewöhnlich dem Verbum unmittelbar folgend ... Dieser Art Sätze weisen eine besondere emotionale Färbung auf".

Erben trata en su gramática los aspectos sintácticos y fonológicos de las partículas, a los que él confiere mayor relevancia. Afirma junto con Krivonosov ${ }^{9}$ „ein Satz mit einer modalen Partikel wird anders gelesen bzw. gesprochen, als derselbe Satz ohne die modale Partikel ". Este aspecto se ha podido comprobar en la introducción donde la diferencia entre ambos textos es clara. La entonación, que no había sido tenida en cuenta hasta ahora en las definiciones, es sin embargo de gran relevancia para aquellos investigadores que sobre todo

\footnotetext{
${ }^{7}$ Helbig/Buscha, Deutsche Grammatik. Ein Handbuch für den Ausländerunterricht, Leipzig, Langenscheidt, 1993, pág. 475.

${ }^{8}$ Erben, J. (1993): Einführung in die deutsche Wortbildungslehre, Berlin: Schmidt, pág. 178

${ }^{9}$ Krivonosov A. (1977a): Die modalen Partikeln in der deutschen Gegenwartssprache, Göppingen: Kümmerle pág. 307
} 
se apoyan en el componente ilocutivo. Erben destaca en el apartado sintáctico, como anteriormente otros investigadores, la posición de las partículas que generalmente debe ser detrás del verbo.

En la gramática de Engel ${ }^{10}$ las partículas modales

„sind auf illokutive Komponente der Äußerung hin orientiert. Zwar verändern sie die Redeabsicht nicht grundlegend sind aber doch in der Lage, sie mehr oder weniger zu modifizieren, zu verstärken, abzumildern ... sind unveränderliche Wörter, die weder allein im Vorfeld des Konstativsatzes stehen noch als Antworten auf irgendwelche Fragen fungieren noch negiert werden können noch häufbar sind“.

Engel hace referencia al elemento ilocutivo tan carácterístico en las partículas. Es de gran importancia, porque a través del acto ilocutivo el hablante pretende causar en el oyente una reacción. Para que este acto se produzca debe previamente existir un acto locutivo, a saber una frase sintáctica y semánticamente correcta. Wunderlich propone un ejemplo que ya es clásico para la aclaración de este fenómeno con la frase Du kannst das Fenster schließen. La frase se puede interpretar como un consejo, aseveración, orden... Con un acto locutivo se puede llegar a la realización de varios actos ilocutivos. En estos casos las partículas modales pueden ayudar a descifrar las intenciones del hablante:

(5a) Du kannst mal das Fenster schließen, una orden

(5b) Du kannst ja das Fenster schließen, un consejo

(5c) Du kannst doch das Fenster schließen, un reproche o una exigencia.

Con este elemento ilocutivo Engel pone de manifiesto la importancia de la pragmática en la interpretación de las partículas. Weydt ya había insistido en el creciente papel que ejerce la pragmática en detrimento de la semántica, porque como dice Monteiro ${ }^{11}$, y como también se ha obsevado en la partícula denn, „...diese Funktionsklasse (die Partikel) lie $\beta$ sich nicht mit rein semantischen Erklärungsformeln der Art 'doch bedeutet $X^{\prime}$ beschreiben".

Por último Duden ${ }^{12}$ ofrece la siguiente definición: „, bestimmte Adverbien werden -vor allem in der gesprochenen Sprache-verwendet, um eine Aussage zu färben und abzutönen". Es quizás el menos explícito y el que menos datos aporta. Sin embargo, al igual que Erben, pone el acento sobre la relevancia del escenario donde tienen lugar la mayoría de los usos de las partículas: el lenguaje coloquial.

Haciendo una sinopsis de las descripciones formuladas por los investigadores se llega a una serie de características relevantes en la descripción y reconocimiento de las partículas:

1. Su presencia afecta a toda la frase y no a un elemento determinado.

\footnotetext{
${ }^{10}$ Engel, U. (1988): Syntax der deutschen Gegenwartssprache, Berlin: Schmidt, pág. 231 y 774

${ }^{11}$ Monteiro, S. (1995): Die Wiedergabe der Abtönungspartikeln doch, ja, eben und halt im Englischen auf der Grundlage literarischer Übersetzungen, Bonn (Tesis doctoral) pág. 20.
}

${ }^{12}$ Duden, K. (1984): Grammatik der deutschen Gegenwartssprache, Mannheim: Dudenverlag, pág. 351 
2. En segundo lugar se encuentran habitualmente en el lenguaje coloquial y en menor medida en el escrito, excepto si se quiere recrear el lenguaje coloquial como puede ser el caso del teatro.

3. En conexión con la anterior, las partículas están orientadas al elemento ilocutivo.

4. Por último decir que sólo tienen un determinado valor o significado dentro de un contexto, es decir, tienen un solo valor dentro de una situación determinada, que no es aplicable a otras frases ni a otros contextos y situaciones diferentes.

Todos estos caracteres conducen más al terreno pragmático, donde esta serie de partículas encuentran un sentido y sobre todo una posible clasificación ${ }^{13}$. Esto último se verá en el siguiente apartado donde el carácter temporal puede ayudar a una mejor comprensión de tan aparentemente intratables partículas.

\section{ANÁLISIS DE ALGUNAS PARTÍCULAS}

Una vez realizado el análisis preceptivo del funcionamiento de las partículas modales el presente artículo se adentra en el tema que acomuna a este encuentro: el tiempo y en este caso, el factor tiempo en las partículas modales alemanas. El carácter temporal de las partículas no ha sido visto como tema de investigación relevante entre los lingüistas. Ello lleva a dos tipos de obsevaciones: o bien no se trata de un aspecto predominante en las partículas y por lo tanto sin interés para el investigador y sin relevancia para su estudio, o bien se pone en duda de que el carácter temporal tenga alguna relación con la modalidad de la frase. El análisis propuesto aquí parte de algunas de las partículas que teóricamente tienen un matiz temporal en algunos de sus significados como así es en denn, eben, erst, gerade, mal, noch, nur, schon. Otras también con matiz temporal como wohl, vielleicht o blo $\beta$ no han sido analizadas por cuestiones de tiempo y espacio.

\section{nur}

Esta partícula tiene asignada dos funciones. Como partícula modal y como partícula lógica. Con el concepto de partícula lógica Krivonosov se refiere a valor semántico. Así se presentan los valores sobre las siguientes frases:

(6a) Wie habe ich nur leben können, ohne dich?

(6b) Raten Sie nur nicht!

(6c) Nur wir beide sind noch da

(6d) Der Mann hatte nur fünf Minuten Zeit

La función de esta partícula es muy semejante a erst, aunque no se puede hablar de sinónimos. En 6a y 6 b el valor pragmático está por encima del semántico. Helbig/Buscha en su gramática dicen sobre esta alternancia de la semántica y pragmática ${ }^{14}$

\footnotetext{
${ }^{13}$ Véase el artículo de Heringer sobre una posible clasificación prototípica de las partículas.
}

14 Helbig/Buscha (1993): Deutsche Grammatik. Ein Handbuch für den Ausländerunterricht, Leipzig, Langenscheidt, pág. 476. 
„Es gibt jedoch Partikeln, deren semantischer Gehalt sehr gering ist, die nahezu ohne eigentliche (denotative)Bedeutung sind, die vielmehr die Anteilnahme des Sprechers bzw. die Art der Sprechhandlung signalisieren, die also mehr kommunikativen als semantischenWert haben".

Con nur el hablante expresa la imposibilidad de la acción que se le requiere en 6a. En $6 \mathrm{~b}$ esta imposibilidad se vuelve prohibición inmediata. Sin embargo en $6 \mathrm{c}$ y $6 \mathrm{~d}$ nur adquiere un carácter restrictivo. El valor temporal aquí no está presente, puesto que no se ve apoyado por ningún complemento de tiempo exceptuando 6d. La traducción de éstas, de mayor contenido semántico, sería de la siguiente manera: Sólo nosotros estamos aquí todavía. El hombre sólo tenía cinco minutos. Su carácter temporal es débil y con su presencia tan sólo refuerzan un complemento temporal al mismo tiempo.

\section{$\underline{\text { denn }}$}

Como se ha visto en la primera parte de este estudio en los ejemplos anteriormente citados, esta partícula puede llegar a realizar tres funciones. Así se desprende de las siguientes frases como objeto de análisis:

(7a) Was ist es denn für einen Wagen?

(7b) Wo vor denn Angst?

(7c) Also, ich gehe denn jetzt

(7d) Ein Mißverständnis ...Denn er tut sowas normalerweise nicht

En 7a y 7b la partícula denn modifica el matiz de la frase, en las que el hablante solicita una explicación de algo que no entiende. En 7c denn tiene un valor temporal en tanto que modifica al verbo. Y en $7 \mathrm{~d}$ se trata de un valor causal. Los tres tipos de partícula cumplen una función sintáctica diferente. De las dos primeras afirma Krivonosov que el valor semántico casi no existe; se trata más bien de una postura del hablante. 7c interesa más desde el punto de vista del tema aquí tratado. El carácter temporal se manifiesta de manera más evidente que en la partícula anterior. Thurmair añade que en esta función adverbial,,.. als die regionale Variante von dann bezeichnet werden kann" $" 15$. El hablante indica con ella cuándo la acción del verbo se puede realizar o no. La traducción en este caso es más sencilla y en esta función se podría traducir por entonces en la frase propuesta.

\section{eben}

A esta partícula aparentemente sin significado y difícil de traducir le son asignada cuatro funciones diferentes por Krivonosov y Thurmair. Como adjetivo se ve en 8a. En esta función no supone ninguna dificultad para el análisis. Como partícula en 8 b. En este caso eben sería una mezcla de ja y denn. En 8c el valor sin embargo sería de un hecho evidente, de una constatación. Como adverbio puede representar el carácter temporal en $8 \mathrm{~d}$. El hablante expresa en primer lugar la certeza de un hecho -en este caso que son las cinco- y en segundo lugar que acaban de dar las cinco en este momento. Se trata de una partícula que recuerda al valor de gerade, aunque ésta contiene un mayor carácter temporal, lo cual no hace factible el test de sustitución de una por otra. Sin embargo en 8 e como reacción a una afirmación, la partícula está carente de cualquier elemento temporal. Esto lleva a pensar

${ }^{15}$ Thurmair, M. (1989): Modalpartikeln und ..., pág. 163. 
en la necesidad de la presencia de un complemento temporal para que se dé esta temporalidad. La traducción de ambas frases sería también diferente: Acaban de dar efectivamente las cinco y Sí, es verdad.

(8a) Das ist eine ebene Fläche

(8b) Ich komme eben morgen

(8c) Amerikaner sind eben so

(8d) Eben hat es fünf Uhr geschlagen

(8e) Ja, eben

\section{$\underline{\text { erst }}$}

Lo que primero llama la atención de esta partícula frente a las demás es su marcado carácter temporal. O al menos es con esta intención con la que generalmante se hace uso de ella. Frases del tipo 9a recuerda al significado de nur. También 9b. El valor de restricción sigue manteniéndose. Sin embargo no es éste el único valor que tiene. Erst puede formar como partícula oraciones desiderativas del tipo 9c o puede funcionar como un adjetivo 9d. Sin embargo como adverbio vuelve a recuperar el carácter temporal 9e. Con este valor puede aparecer la forma erstmal. En cuanto a la traducción a veces se puede traducir, y a veces no se debe traducir. En esos casos refuerza la acción. Sólo tiene 20 años. Mañana llega mi hermana. ¡Ojalá viniera!. Es la primera y última vez. Primero queremos comer.

(9a) Er ist erst 20 Jahre alt

(9b) Erst morgen kommt meine Schwester

(9c) Wenn er erst Käme!

(9d) Es ist das erste und letzte Mal!

(9e) Erst wollen wir mal essen

\section{gerade}

También el uso de esta partícula se conoce más bien con carácter temporal. Obsérvese entonces cuáles son los valores. En 10a el hablante insiste en que la acción acaba de ser realizada. Es decir, indica inmediatez en la realización de la acción. Otros valores de gerade se reflejan en las siguientes frases $10 \mathrm{~b}$. Aquí como partícula lógica, valor semántico, hace referencia a un miembro de la frase y tiene un valor de insistencia, que en este caso se refiere al sujeto de la frase. Se podría comparar semánticamente a ausgerechnet. En castellano: Precisamente él tiene que repetir.

(10a) Das habe ich gerade gemacht

(10b) Gerade er muß nachholen

\section{$\underline{\text { mal }}$}

Se trata de una partícula que en la lengua hablada se utiliza con mucha frecuencia. Se supone que es una reducción de la forma einmal. También esta partícula puede funcionar de varias maneras. La más frecuente es junto a una forma de imperativo (11a). Aquí forma con el verbo un núcleo sintáctico y fonológico. En algunos casos se utiliza la partícula de manera sistemática sobre todo en compañía de una forma verbal monosílaba como $11 \mathrm{~b}$. Incluso no sólo fonológica, sino también gráficamente puede formar una unidad. El hablante no está indicando nada nuevo, tan sólo, como dice Thurmair, se suaviza la orden del imperativo generalmente en un discurso oral. Mal tiene otra función como adverbio. En 
esta función se le conoce un valor temporal. Sólo recordar como ejemplo el comienzo de los cuentos en alemán (11c). O también en 11d. Mal expresa una fecha vaga sin ser precisada. La traducción podría equivaler a alguna vez he visto a ese hombre.
(11a) Ruf ihn mal an! Probier's mal!
(11b) Sagmal, Hörmal, Ißmal
(11c) Es war einmal ein alter Mann
(11d) Ich habe ihn mal gesehen

\section{noch}

Esta partícula vuelve a ser problemática entre los críticos. No está muy clara su condición de partícula. Helbig/Buscha y Krivonosov sin embargo la consideran como tal. Noch tiene la facultad de poder aparecer en combinación con otras partículas para intensificar la frase como se da en 12a. Este uso junto con el comparativo es muy frecuente y recurrente. La duración de una acción es otra característica de esta partícula como en 12b. Si se añadiera fünf Minuten noch esta duración se limitaría. Sin embargo introduciendo un complemento directo como en la frase Er hat nóch eine Fráge indica que la acción no ha terminado y por tanto continúa. La diferencia sería de tipo sintáctico. En er arbeitet noch la partícula afecta al verbo y en $12 \mathrm{c}$ afecta al complemento.

(12a) Die Arbeit war noch schwieriger

(12b) Er arbeitet noch (fünf Minuten)

(12c) Er hat nóch eine Fráge

\section{schon}

La partícula schon no ofrece dudas sobre su pertenencia a esta clase de palabras, aunque sí es difícil su clasificación. 13a y 13b se diferencian de 13c claramente en que en la última frase se podría prescindir de schon. En 13a se trata de una frase cuyo elemento temporal proporciona el significado final casi aspectual. Sin embargo sin él ese elemento de inesperado no existiría. 13b se diferencia de 13a en que está exenta del elemento temporal y la partícula pasa a transformarse en afirmación del contenido.

(13a) Ihr seid schon da?

(13b) Die Stadt Reutte liegt schon in Tirol

(13c) Wer verliert schon gern beim Spiel?

\section{CONCLUSIÓN}

Los análisis de las partículas han evidenciado netamente que los respectivos significados y las funciones pragmáticas de las partículas son muy variables y fuertemente orientadas al elemento ilocutivo, y dependientes del contexto. Este aspecto es común a todas las partículas analizadas. Pero sin embargo -y con ello se responde a la pregunta planteada al inicio- hay algo común a todas ellas. Todas las partículas aquí analizadas tienen una mayor o menor afinidad temporal. Esta observación acerca de la presencia de temporalidad en las partículas modales parece abrir nuevas perspectivas sobre los problemas de estas partículas tan carcterísticas de la lengua alemana.

La variedad de significados aumenta en tanto que las partículas son analizadas en el nivel textual o pragmático. Esta enorme variación explica que a los estudiantes de lengua 
alemena se les diga con frecuencia que estas partículas no poseen ningún significado. Es sin duda más sencillo y menos traumático. Sin embargo la realidad refleja un paisaje muy distinto. La prueba de sustitución muestra que casi no es concebible un texto sin partículas. Las partículas tienen un significado, y a veces en una polisemia tan alta que sólo por medio de la presencia del hablante en un lugar y tiempo determinado puede deshacerse la ambigüedad que provocan. Este hecho explica porqué una clasificación y sistematización de estas partículas hasta ahora apenas ha sido posible. El criterio de la temporalidad puede ser eventualmente una ayuda fructífera. Si se parte en el sentido de una semántica de los prototipos de la existencia de un núcleo de significado, es posible crear dos grupos:

1. Un primer grupo formado por las partículas eben, erst, gerade, schon, noch, cuyo núcleo de significado es temporal y tienen muchos significados secundarios modales.

2. Y un segundo grupo formado por nur, mal, denn, cuyo núcleo de significado es modal, pero también con valores secundarios temporales.

La conclusión de este análisis acerca de la temporalidad de las partículas no hace más que constatar la necesidad de un estudio más profundo de las partículas modales en nuevos marcos con el fin de evitar en el futuro comentarios como los de L. Reiners sobre las partículas modales (1951:159): „Alle diese Flickworte wimmeln wie Läuse im Pelz unserer Sprache herum“ „Beiwörter, die nur verzieren,..., müssen wir streichen.“.

\section{BIBLIOGRAFÍA}

DUDEN, K. (1984): Grammatik der deutschen Gegenwartssprache, Mannheim: Dudenverlag.

ENGEL, U. (1982): Syntax der deutschen Gegenwartssprache, Berlin: Schmidt.

ERBEN, J. (1993): Einführung in die deutsche Wortbildungslehre, Berlin: Schmidt.

HELBIG, G./ BUSCHA, J. (1993): Deutsche Grammatik, Leipzig: Langenscheidt.

KRIVONOSOV, A. (1965b): „Wechselbeziehungen zwischen den modalen Partikeln und der Satzintonation im Deutschen", en: Zeitschrift für Phonetik, Sprachwissenschaft und Kommunikationsforschung, 18.

KRIVONOSOV, A. (1977a): Die modalen Partikeln in der deutschen Gegenwartssprache, Göppingen: Kümmerle.

LORENZO, G. (2001): Comprender a Chomsky, Madrid: Antonio Machado.

MONTEIRO, S. (1995): Die Wiedergabe der Abtönungspartikeln doch, ja, eben und halt im Englischen auf der Grundlage literarischer Übersetzungen, Bonn (Tesis doctoral).

SCHRÖDER, G. (1965): “Zu einigen bedeutungsgeminderten Adverbien (Wurzwörtern)“, en: Deutsch als Fremdsprache, 1, Leipzig, Herder-Institut.

THURMAIR, M. (1989), Modalpartikeln und ihre Kombinationen, Tübingen: Niemeyer.

WAHRIG, G. (1991): Deutsches Wörterbuch, München: Bertelsmann. 
WEYDT, H. (1969): Abtönungspartikel. Die deutschen Modalwörter und ihre französische Entsprechungen, Bad Homburg: Gehlen.

Wunderlich, D. (1976): Studien zur Sprechakttheorie, Frankfurt am Main: Suhrkamp.

ZYBATOW, L. (1990): Was die Partikeln bedeuten: eine konstrastive Analyse Russisch-Deutsch, München: Sagner. 